\title{
Print exposure modulates the effects of repetition priming during sentence reading
}

\author{
Matthew W. Lowder ${ }^{1}$ - Peter C. Gordon ${ }^{2}$
}

Published online: 21 February 2017

(C) Psychonomic Society, Inc. 2017

\begin{abstract}
Individual readers vary greatly in the quality of their lexical representations, and consequently in how quickly and efficiently they can access orthographic and lexical knowledge. This variability may be explained, at least in part, by individual differences in exposure to printed language, because practice at reading promotes the development of stronger reading skills. In the present eyetracking experiment, we tested the hypothesis that the efficiency of word recognition during reading improves with increases in print exposure, by determining whether the magnitude of the repetition-priming effect is modulated by individual differences in scores on the author recognition test (ART). Lexical repetition of target words was manipulated across pairs of unrelated sentences that were presented on consecutive trials. The magnitude of the repetition effect was modulated by print exposure in early measures of processing, such that the magnitude of the effect was inversely related to scores on the ART. The results showed that low levels of print exposure, and thus lowerquality lexical representations, are associated with high levels of difficulty recognizing words, and thus with the greatest room to benefit from repetition. Furthermore, the interaction between scores on the ART and repetition suggests that print exposure is not simply an index of general reading speed, but rather that higher levels of print exposure are associated with an enhanced ability to access lexical knowledge and recognize words during reading.
\end{abstract}

Matthew W. Lowder

mlowder@ucdavis.edu

1 Department of Psychology, University of California, Davis, Young Hall, One Shields Ave., Davis, CA 95616, USA

2 Department of Psychology, University of North Carolina, Chapel Hill, NC, USA
Keywords Repetition priming · Eye movements · Reading · Visual word recognition

Efforts to understand the nature of word recognition during reading have traditionally focused on the characteristics of words or texts that reliably modulate the targeting of saccades or the duration of fixations. For example, a large literature has demonstrated that words that are short, frequent, and predictable are skipped more often and elicit shorter fixation durations than do words that are long, infrequent, and unpredictable (for reviews, see Clifton et al., 2016; Rayner, 1978, 1998). Although a great deal has been learned about the text-level characteristics that contribute to the relative ease or difficulty of recognizing words during reading, considerably less work has been directed at understanding how variability in cognitive constructs among individual readers contributes to differences in word recognition. Indeed, a complete understanding of the cognitive mechanisms underlying word recognition during reading must not only be able to account for the lexical factors that contribute to this process, but must also account for systematic differences in word recognition among individual readers, including how text-level and individual-level factors interact.

One approach that has proven quite useful in explaining individual differences in reading and language processing involves estimating an individual's level of print exposure, most commonly by administering a recognition test of authors' names. In the author recognition test (ART), originally developed by Stanovich and West (1989), participants are presented with a checklist of author and nonauthor names and are instructed to mark the names they recognize as authors. The underlying assumption is that individuals who read frequently are more likely to have encountered the names of authors than are those who read less frequently. The ART shows moderate- 
to-strong relationships with other measures of verbal ability, including vocabulary knowledge (Beech, 2002; Lewellen, Goldinger, Pisoni, \& Greene, 1993; Stanovich, West, \& Harrison, 1995), reading comprehension (Martin-Chang \& Gould, 2008; Stanovich \& Cunningham, 1992), knowledge of spelling (Lewellen et al., 1993; Stanovich \& West, 1989), and standardized tests of verbal ability (Acheson, Wells, \& MacDonald, 2008; Lewellen et al., 1993; Stanovich et al., 1995). Furthermore, a meta-analysis of studies examining the contribution of print exposure to language development from infancy to early adulthood suggests a causal interdependence among time spent reading, reading skill, and reading enjoyment (Mol \& Bus, 2011). That is, more time spent reading (or being read to) at a young age leads to early advancements in reading skill, and thus enhanced reading enjoyment, which in turn leads to more time spent reading, and so on. The idea that practice at reading is a key factor that contributes to reading skill is broadly consistent with Perfetti's lexical-quality hypothesis (Perfetti, 1985, 2007), which proposes that readers with high levels of linguistic expertise acquire highquality linguistic representations that allow for low-level knowledge of orthographic forms to be automatically linked to higher-level semantic knowledge, thus contributing to rapid and efficient word recognition during reading.

Previous work examining the relationship between print exposure and word recognition has primarily relied on single-word presentation paradigms, as in the lexicaldecision task (Chateau \& Jared, 2000; Lewellen et al., 1993; Sears, Campbell, \& Lupker, 2006; Sears, Siakaluk, Chow, \& Buchanan, 2008; Unsworth \& Pexman, 2003). This work has generally shown that not only do individuals with higher levels of print exposure make faster and more accurate lexical decisions, but they also access lexical information more efficiently, as revealed by smaller word-frequency effects than among individuals with lower levels of print exposure. More recent studies have begun to relate performance on the ART to measures of eye-movement behavior during reading. For example, Moore and Gordon (2015) showed that higher ART scores were associated with shorter gaze durations (i.e., the first-pass reading time on a word), as well as reduced wordfrequency effects. In addition, Choi, Lowder, Ferreira, and Henderson (2015) used a gaze-contingent moving-window paradigm to show that higher ART scores (as well as higher scores on other measures of verbal ability) were associated with larger perceptual span during reading. That is, readers with higher levels of print exposure were more efficient at extracting parafoveal information from the right of the currently fixated word.

In the present experiment, we tested the hypothesis that the efficiency of word recognition during reading improves with increases in print exposure, by determining whether the magnitude of the repetition-priming effect is modulated by individual differences in ART scores. Within the context of word recognition, repetition priming refers to facilitation in the processing of a word when that word has been encountered previously. Although repetition-priming effects have traditionally been studied within the context of list-learning paradigms (e.g., Jacoby \& Dallas, 1981; Scarborough, Cortese, \& Scarborough, 1977; Tulving \& Schacter, 1990) or maskedpriming word-identification paradigms (e.g., Bodner \& Masson, 2001; Forster \& Davis, 1984, 1991), a growing body of work in the eye-movement literature has demonstrated that the repetition of a word facilitates processing during the natural reading of text (Choi \& Gordon, 2013; Gordon, Plummer, \& Choi, 2013; Ledoux, Gordon, Camblin, \& Swaab, 2007; Liversedge, Pickering, Clayes, \& Branigan, 2003; Lowder, Choi, \& Gordon, 2013; Traxler, Foss, Seely, Kaup, \& Morris, 2000). This facilitation has been explained as resulting from enhanced lexical retrieval processes, because repetition effects have been shown to be larger for words that are more difficult to access. For example, Lowder et al. demonstrated enhanced repetition priming for low-frequency as compared to high-frequency words, suggesting that repetition aids the process of lexical access such that the more-difficult-to-find, low-frequency words benefit more from repetition than do the easier-to-find, high-frequency words.

Our study's focus on eyetracking methodology and print exposure offers several advantages over previous investigations of individual differences in visual word recognition. First, most of the previous work examining individual differences in priming effects has relied on the lexical-decision task (e.g., Andrews \& Hersch, 2010; Andrews \& Lo, 2012; Tan \& Yap, 2016; Yap, Tse, \& Balota, 2009), in which participants are required to make a metalinguistic judgment about the target stimulus. Thus, performance on this task inherently reflects decision-related processes in addition to the more theoretically relevant process of lexical retrieval. In contrast, the eyetracking paradigm does not require any explicit judgment about the target word, and thus has the advantage of more accurately reflecting wordrecognition processes as they occur during reading. Second, previous work has tended to focus primarily on individual differences related to vocabulary knowledge and spelling ability and has not considered the role of print exposure. In particular, Andrews and colleagues (Andrews \& Hersh, 2010; Andrews \& Lo, 2012, 2013) have argued that spelling ability is a particularly powerful construct for indexing individual differences in lexical quality. Although this may be true, the bulk of this evidence has come from experiments that have related performance on spelling tests to performance on the lexical-decision task, which is potentially problematic, given that the lexical-decision task itself can be viewed as a type of spelling test. In contrast, the task demands reflected by the ART (i.e., to identify author names) and the eyetracking task (i.e., to read naturally) are conceptually distinct from one another. Finally, the design of the present experiment contributes to the important goal of replicating key findings across a range of different methodological approaches, 
by testing whether the individual differences in priming effects that have been reported in lexical-decision paradigms will also emerge in an eyetracking paradigm.

In the present eyetracking-while-reading experiment, we systematically manipulated whether a target word was repeated or new across pairs of unrelated sentences and also collected ART scores from each of our participants. If readers with low print exposure have lower-quality lexical representations than readers with high print exposure, they should also experience greater difficulty accessing words during reading. For that reason, readers with low print exposure should have a greater capacity to benefit from lexical repetition, and should therefore show larger repetition-priming effects.

\section{Method}

\section{Participants}

Forty-eight students at the University of North Carolina at Chapel Hill participated in this experiment in exchange for course credit. They were all native English speakers and reported normal or corrected-to-normal vision.

\section{Materials and design}

Sixteen pairs of sentences were constructed (see Example 1). The first of each pair served as the prime-bearing sentence (1a), whereas the second served as the target-bearing sentence (1b). Thirty-two proper names were chosen to serve as the prime and target words. Proper names were used because they can easily be placed in different sentence positions without the lexical-semantic constraints associated with content words, and because they can be counterbalanced across prime and target positions. In addition, previous work has indicated that the initial stages of word recognition operate similarly for proper names and common nouns (Valentine, Bredart, Lawson, \& Ward, 1991; Valentine, Moore, Flude, Young, \& Ellis, 1993; Van Petten, Kutas, Kluender, Mitchiner, \& McIsaac, 1991). Finally, our own previous work has demonstrated robust repetition-priming effects with proper names (Gordon et al., 2013; Ledoux et al., 2007; Lowder et al., 2013). The names that were chosen were drawn from a database containing the names of all first-year undergraduate students who had entered UNC over a 5-year period. All of the names were low-frequency, defined as appearing in the corpus only four or five times. The names were between five and eight characters in length. Two names of the same length and gender were assigned to each pair of sentences, and different versions of the sentences were created by allowing each name to alternately serve as prime and target in the repeated and new conditions (see Example 1). One version of each item was assigned to one of four counterbalanced lists, such that no participant saw more than one version of each item, and such that all participants received 16 prime-bearing and 16 targetbearing sentences, with half containing a repeated target and half containing a new target. In addition, there were 124 filler sentences representing a variety of structures. Every sentence was followed by a true-or-false comprehension question. The comprehension question that followed an experimental item never contained a proper name.

(1a) Prime: The TV was on all night even though [Selena/ Blythe] had fallen asleep very early.

(1b) Target: Yesterday morning, I made sure to thank [Selena/Blythe] for the Christmas gift.

\section{Author recognition test}

Participants completed a recently updated version of the ART (Moore \& Gordon, 2015). The test consists of a list of 130 names: 65 are the names of real authors, and 65 are names that do not refer to authors (foils). The names are presented in alphabetical order. Participants are instructed to mark only the names that they know to be authors, and they are told that they will be penalized for marking nonauthors. The score is calculated as the number of authors correctly selected minus the number of foils incorrectly selected.

\section{Procedure}

Eye movements were recorded with an EyeLink 1000 system (SR Research, Ottawa, ON), which was calibrated at the beginning of each session and recalibrated throughout the session as needed. A headrest and chinrest were used to minimize head movement. At the start of each trial, a fixation point was presented near the left edge of the computer screen. Once the participant's gaze was steady on this point, the experimenter pressed a button that triggered sentence presentation. After reading the sentence, the participant pressed a button on a handheld console, which replaced the sentence with a truefalse comprehension question. Participants pressed one button to answer "true" and a different button to answer "false." The mean comprehension question accuracy was $96 \%$. After the participant had answered the question, the fixation point for the next trial appeared.

Participants were first presented with four of the filler sentences. After this warmup block, the remaining sentences were presented in a different random order for each participant, while preserving the critical order of the prime-target pairs. In other words, each prime sentence was always followed immediately by its corresponding target sentence (with an intervening comprehension question). Following the eyetracking portion of the experiment, participants completed the ART. 


\section{Analysis}

Analysis of the eyetracking data focused on seven standard eye-movement measures that reflect a range of processing stages (Rayner, 1998). Skipping rate is the proportion of trials on which a target word is not fixated at all or is only fixated after a subsequent word has been fixated. Single-fixation duration is the average across trials of the durations of the initial first-pass fixations on a word, provided that the word received only one first-pass fixation. First-fixation duration is the average of the durations of the initial first-pass fixations on a word, regardless of the total number of first-pass fixations. Gaze duration is the average of the sum of all first-pass fixations on a word. These four measures are thought to reflect the earliest stages of word recognition, including processes of perceptual encoding and lexical access. First-pass regression rate is the proportion of trials on which a first-pass fixation on a word is followed by a regressive rather than a progressive saccade. Regression-path duration (also called go-past time) is the sum of all fixations beginning with the initial fixation on a word and ending when gaze is directed away from the region to the right. Thus, regression-path duration includes time spent rereading earlier parts of the sentence, before the reader is ready to move to the right of the target word. first-pass regression rate and regression-path duration are thought to reflect processes involved in integrating a word with earlier parts of the sentence. Rereading duration is the sum of all fixations on a word that are not included in gaze duration. Unlike the other measures, rereading duration contains zeroes (i.e., trials on which the word was not refixated after first-pass reading). This measure is thought to reflect later stages of processing, including any lingering difficulty associated with integrating the word with the rest of the sentence.

An automatic procedure in the EyeLink software combined fixations that were shorter than $80 \mathrm{~ms}$ and that were within one character of another fixation into a single fixation. Additional fixations shorter than $80 \mathrm{~ms}$ or longer than 1,000 ms were eliminated. In addition, means and standard deviations were computed separately for each dependent measure within the repeated and new conditions. Reading times that were greater than 2.5 standard deviations from the condition mean were eliminated. This procedure affected $2.6 \%$ of the data.

The data were analyzed using mixed-effects models in the lme4 package (Bates, Maechler, \& Bolker, 2012) in R. Fixed effects included the experimental factors Repetition (new vs. repeated) and ART Score (mean-centered), as well as the repetition-by-ART interaction. Subjects and items were entered as crossed random effects, including maximally appropriate random intercepts and slopes. In cases in which the model failed to converge, the random-effects structure was sequentially simplified until convergence was achieved (Barr, Levy, Scheepers, \& Tily, 2013). For the fixation duration measures (single-fixation duration, first-fixation duration, gaze duration, regression-path duration, and rereading duration), linear mixed-effects regression models were fit using the lmer function, which provides the regression coefficient $(b)$, standard error $(S E)$, and $t$ value of the coefficient. For the fixation probability measures (skipping rate and first-pass regression rate), logistic mixed-effects regression models were fit using the glmer function, which provides the regression coefficient, standard error, and $z$ value of the coefficient. Effects were considered significant when the absolute value of $t$ or $z$ was 2 or greater.

\section{Results}

\section{ART scores}

ART scores varied substantially across participants, with a minimum score of 1 and a maximum score of 44 . The mean was 16.73, the standard deviation was 9.91, and the median was 14 .

\section{Eye movements}

Mean values for all eye-movement measures are presented in Table 1 . The results of the mixed-effects analyses are presented in Tables 2 and 3. Main effects of repetition were observed in the measures of single-fixation duration, gaze duration, regression-path duration, and first-pass regression rates, such that repeated targets elicited shorter reading times and fewer regressions than did new targets. Main effects of ART score

Table 1 Mean eye-movement measures on the target word as a function of repetition condition

\begin{tabular}{|c|c|c|c|c|c|c|c|}
\hline \multirow[t]{2}{*}{ Condition } & \multicolumn{5}{|c|}{ Fixation duration measures (ms) } & \multicolumn{2}{|c|}{ Probability measures } \\
\hline & SFD & FFD & GZD & RPD & Rereading & Skipping & Regressions \\
\hline New & 214 & 214 & 246 & 310 & 111 & .18 & .19 \\
\hline Repeated & 200 & 201 & 221 & 247 & 100 & .21 & .10 \\
\hline
\end{tabular}

$S F D$ single-fixation duration, $F F D$ first-fixation duration, $G Z D$ gaze duration, $R P D$ regression-path duration 
Table 2 Results of mixed-effects analyses for the fixation duration measures

\begin{tabular}{|c|c|c|c|c|c|c|c|c|c|c|c|c|c|c|c|}
\hline \multirow[t]{2}{*}{ Parameters } & \multicolumn{3}{|l|}{ SFD } & \multicolumn{3}{|l|}{ FFD } & \multicolumn{3}{|l|}{ GZD } & \multicolumn{3}{|l|}{ RPD } & \multicolumn{3}{|c|}{ Rereading } \\
\hline & $b$ & $S E$ & $t$ & $b$ & $S E$ & $t$ & $b$ & $S E$ & $t$ & $b$ & $S E$ & $t$ & $b$ & $S E$ & $t$ \\
\hline (Intercept) & 210.21 & 4.68 & 44.90 & 209.32 & 4.74 & 44.21 & 273.13 & 6.54 & 36.27 & 300.42 & 15.46 & 19.43 & 103.24 & 12.39 & 8.33 \\
\hline Repetition & -11.18 & 5.21 & -2.15 & -9.61 & 5.06 & -1.90 & -20.84 & 7.54 & -2.76 & -59.06 & 13.81 & -4.28 & -13.53 & 15.46 & -0.88 \\
\hline ART & -22.97 & 6.36 & -3.61 & -21.44 & 6.24 & -3.43 & -33.67 & 8.60 & -3.92 & -42.15 & 15.56 & -2.71 & -12.44 & 17.51 & -0.71 \\
\hline Repetition $\times$ ART & 17.59 & 6.09 & 2.89 & 12.30 & 5.55 & 2.22 & 14.88 & 7.23 & 2.06 & 19.25 & 14.23 & 1.35 & -3.51 & 15.93 & -0.22 \\
\hline
\end{tabular}

$A R T$ author recognition test, $S F D$ single-fixation duration $F F D$ first-fixation duration, GZD gaze duration, $R P D$ regression-path duration. Statistically significant effects are indicated in boldface

were observed in the measures of single-fixation duration, first-fixation duration, gaze duration, and regression-path duration, with higher levels of print exposure being associated with shorter reading times. In addition to these main effects, we observed significant repetition-by-ART interactions in measures of single-fixation duration, first-fixation duration, and gaze duration. These effects are depicted graphically in Fig. 1, with ART scores binned into thirds for purposes of illustration. For all three measures, the effect of repetition was robust for readers with lower ART scores and became smaller as ART score increased, such that for the highest ART scorers, the effect was absent altogether.

\section{Discussion}

The research reported here combined eye-movement measures from a repetition-priming paradigm and participants' scores on the author recognition test to determine whether the efficiency of word recognition during reading improves with increases in print exposure. The results showed significant repetition effects in eyetracking measures associated with both early stages of lexical access (single-fixation duration, gaze duration) and later stages of integration (regression-path duration, first-pass regressions). In addition, higher ART scores were associated with shorter reading times in measures

Table 3 Results of mixed-effects analyses for the fixation probability measures

\begin{tabular}{lllllllll}
\hline Parameters & \multicolumn{2}{l}{ Skipping } & & & \multicolumn{2}{l}{ Regressions } \\
\cline { 2 - 4 } \cline { 7 - 9 } & $b$ & $S E$ & $z$ & & $b$ & $S E$ & $z$ \\
\hline Intercept) & -1.81 & 0.23 & $\mathbf{- 7 . 8 5}$ & & -1.53 & 0.17 & $\mathbf{- 9 . 1 3}$ \\
Repetition & 0.19 & 0.19 & 0.97 & -0.76 & 0.25 & $\mathbf{- 3 . 0 7}$ \\
ART & 0.09 & 0.20 & 0.46 & -0.07 & 0.16 & -0.41 \\
Repetition $\times$ ART & 0.27 & 0.20 & 1.37 & & -0.04 & 0.26 & -0.15
\end{tabular}

$A R T$ author recognition test. Statistically significant effects are indicated in boldface of single-fixation duration, first-fixation duration, gaze duration, and regression-path duration. Crucially, the magnitude of the repetition effect was modulated by print exposure in the measures of single-fixation duration, first-fixation duration, and gaze duration, such that the repetition effect was robust for readers with lower ART scores, and absent altogether for readers with higher ART scores. The results have implications for research on individual differences in reading and sentence processing, as well as for the basic mechanisms underlying word recognition during reading.

The ART is generally considered a reliable estimate of an individual's exposure to printed language (Stanovich \& West, 1989), and therefore serves as a good indicator of how much reading practice an individual has had (see Mol \& Bus, 2011; Moore \& Gordon, 2015). Reading practice, then, is believed to promote high-quality linguistic representations that serve to strengthen the associations between low-level knowledge of word forms and higher-level knowledge of word meanings (Perfetti, 1985, 2007). The present results are consistent with this idea, in showing that readers with lower levels of print exposure display an enhanced capacity to benefit from lexical repetition in measures that reflect the earliest stages of word recognition. Thus, the pattern suggests that greater amounts of reading practice, as reflected in higher scores on the ART, are associated with high-quality linguistic representations that support rapid and efficient word recognition during reading. Furthermore, the results highlight the ART as a useful measure for better understanding individual variability in reading and language processing (see also Choi et al., 2015; Moore \& Gordon, 2015). That is, the ART is not simply an index of reading speed, but rather, higher scores on the ART are associated with an enhanced ability to access lexical knowledge during reading.

The results also add to previous demonstrations of repetition priming during the natural reading of a text (Choi \& Gordon, 2013; Gordon et al., 2013; Ledoux et al., 2007; Liversedge et al., 2003; Lowder et al., 2013; Traxler et al., 2000) and provide new insights into the mechanisms underlying these effects. As we have argued previously (see Lowder et al., 2013), the finding that repetition-priming effects during 
a
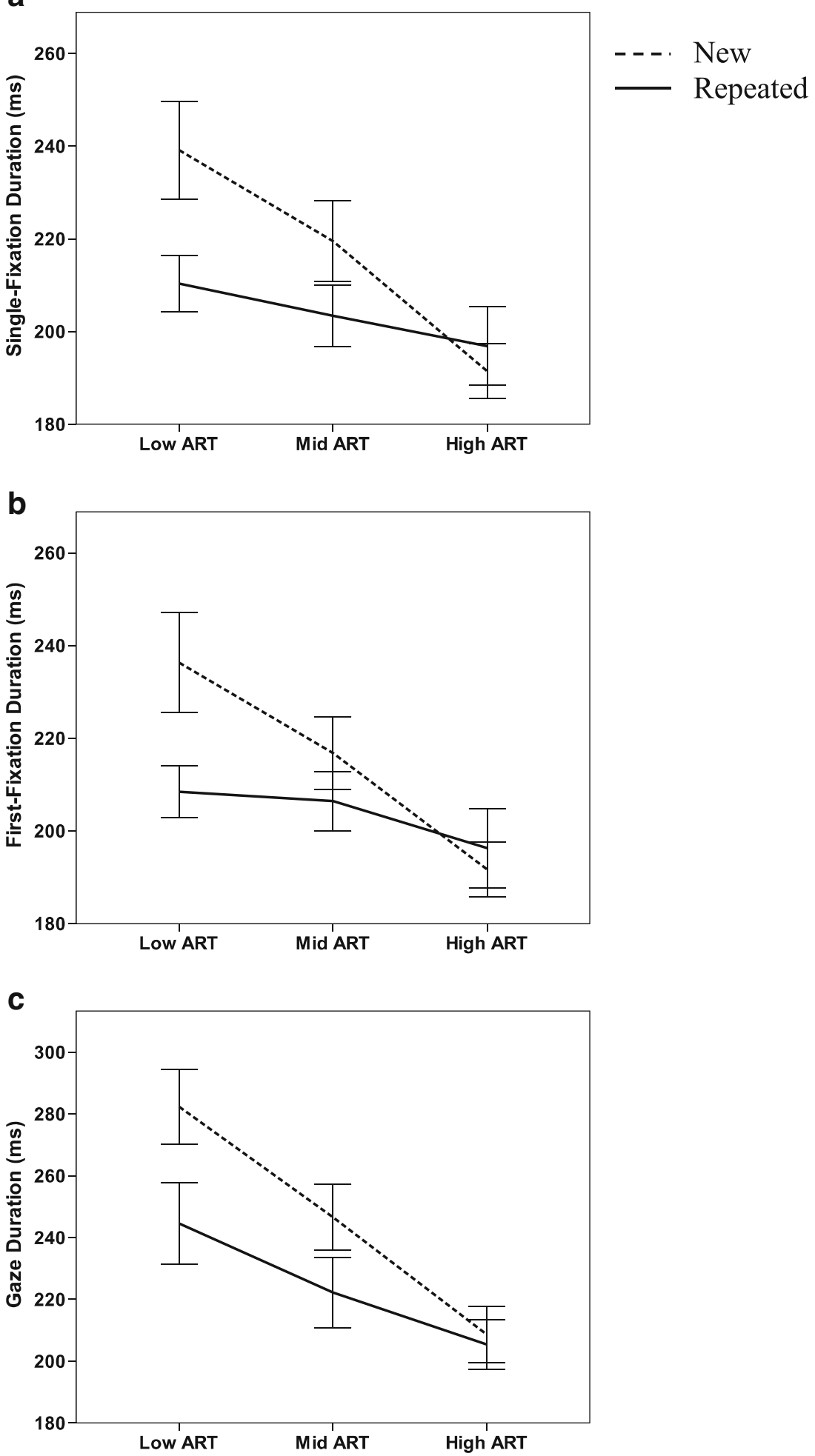

Fig. 1 Mean single-fixation durations (a), first-fixation durations (b), and gaze durations (c) for repeated and new targets as a function of author recognition test (ART) group. ART scores are binned in thirds:

reading persist even when several words intervene between the prime and the target suggests that these effects do not stem from the residual activation of orthographic or phonological
"Low ART" includes scores of 10 or less, "Mid ART" includes scores between 11 and 18, and "High ART" includes scores of 19 and above. Error bars represent standard errors of the means

representations between the prime and target, as has been posited to explain repetition priming in masked word identification paradigms (see Forster \& Davis, 1984; Grainger \& 
Jacobs, 1996). Importantly, the present experiment further suggests that repetition-priming effects during reading do not stem from higher levels of discourse interpretation. Whereas previous reading studies have demonstrated repetition priming with prime and target words inserted in the same sentence, the present experiment has shown evidence of repetition priming across pairs of unrelated sentences that could not be interpreted together as part of a coherent message. This makes it difficult to explain our effects in terms of facilitation at the discourse-level representation. Instead, the results are most readily explained as resulting from enhanced lexicalretrieval processes.

Some have argued that repetition-priming effects are best explained in terms of episodic-memory mechanisms (e.g., Bodner \& Masson, 2001). According to this account, the initial encounter with the prime creates a representation in episodic memory that is then retrieved to facilitate processing of the target. This might suggest, then, that readers with lower levels of print exposure benefited more from lexical repetition because they were more likely than readers with higher levels of print exposure to rely on episodic memory. However, given that the first-pass eyetracking measures we reported are thought to reflect the earliest stages of word recognition rather than processes related to episodic memory (see, e.g., Clifton, Staub, \& Rayner, 2007), we do not believe a memory-based account can explain our pattern of results. This highlights an advantage of eyetracking in the present study as opposed to the lexical-decision task used in most word-recognition priming studies. The first-pass reading-time measures that showed significant repetition-by-ART interactions ranged between 200 and $250 \mathrm{~ms}$ (see Table 1), whereas lexical-decision times tend to range between 500 and $600 \mathrm{~ms}$ (e.g., Andrews \& Lo, 2013; Tan \& Yap, 2016; see Hoedemaker \& Gordon, 2014, 2016, for a discussion of how the long response times in manual lexical-decision tasks affect patterns of semantic priming). The much shorter durations of eyetracking measures as compared to lexical-decision latencies supports our assertion that the repetition effects that we observed reflect lexicalretrieval processes rather than mechanisms related to episodic memory.

Methodological differences between eyetracking and lexical decision might also explain an apparent inconsistency between our results and those of Tan and Yap (2016). Tan and Yap showed an interaction between masked repetition-priming effects and verbal ability (measured by vocabulary and spelling tests), such that the magnitude of the repetition-priming effect was larger for participants who scored higher on these measures of verbal skill. In their experiment (and other experiments like it), the prime was presented very briefly ( $40 \mathrm{~ms})$, making processing of the prime difficult, whereas the target was presented until a response was made. This suggests that higher levels of verbal ability in a masked-priming experiment are helpful for efficiently detecting a rapidly presented prime stimulus, which aids subsequent processing of the target. In contrast, lower levels of verbal ability in the present eyetracking experiment were associated with a greater degree of difficulty recognizing words during natural reading, and thus repetition of a word was most likely to be beneficial for these readers.

In conclusion, this work represents an important step toward understanding how variability in print exposure contributes to basic processes of word recognition during reading. These results also support the notion that repetition-priming effects during reading reflect facilitation in lexical search, rather than facilitation at the level of perceptual encoding or discourse interpretation. Whereas our previous work showed that the words that are most difficult to access are the ones that benefit most from repetition (Lowder et al., 2013), the present work has shown that the individual readers who find it most difficult to access words during reading are the ones who benefit most from repetition.

Author note This research was supported in part by grants from the National Institute of Child Health and Human Development, awarded to M.W.L. (F32 HD084100) and P.C.G. (R01 HD060440-06A2). We thank Rachael Jones for assistance in conducting the experiment.

\section{References}

Acheson, D. J., Wells, J. B., \& MacDonald, M. C. (2008). New and updated tests of print exposure and reading abilities in college students. Behavior Research Methods, 40, 278-289. doi:10.3758/ BRM.40.1.278

Andrews, S., \& Hersch, J. (2010). Lexical precision in skilled readers: Individual differences in masked neighbor priming. Journal of Experimental Psychology: General, 139, 299-318.

Andrews, S., \& Lo, S. (2012). Not all skilled readers have cracked the code: Individual differences in masked form priming. Journal of Experimental Psychology: Learning, Memory, and Cognition, 38, $152-163$

Andrews, S., \& Lo, S. (2013). Is morphological priming stronger for transparent than opaque words? It depends on individual differences in spelling and vocabulary. Journal of Memory and Language, 68, 279-296.

Barr, D. J., Levy, R., Scheepers, C., \& Tily, H. J. (2013). Random effects structure for confirmatory hypothesis testing: Keep it maximal. Journal of Memory and Language, 68, 255-278. doi:10.1016/j. jml.2012.11.001

Bates, D., Maechler, M., \& Bolker, B. (2012). lme4: Linear mixed-effects models using S4 classes [Software]. Retrieved from http://lme4.rforge.r-project/org

Beech, J. R. (2002). Individual differences in mature readers in reading, spelling, and grapheme-phoneme conversion. Current Psychology, 21, 121-132.

Bodner, G. E., \& Masson, M. E. J. (2001). Prime validity affects masked repetition priming: Evidence for an episodic resource account of priming. Journal of Memory and Language, 45, 616-647.

Chateau, D., \& Jared, D. (2000). Exposure to print and word recognition process. Memory \& Cognition, 28, 143-153. doi:10.3758/ BF03211582

Choi, W., \& Gordon, P. C. (2013). Coordination of word recognition and oculomotor control during reading: The role of implicit lexical 
decisions. Journal of Experimental Psychology: Human Perception and Performance, 39, 1032-1046.

Choi, W., Lowder, M. W., Ferreira, F., \& Henderson, J. M. (2015). Individual differences in the perceptual span during reading: Evidence from the moving window technique. Attention, Perception, \& Psychophysics, 77, 2463-2475. doi:10.3758/ s13414-015-0942-1

Clifton, C., Jr., Ferreira, F., Henderson, J. M., Inhoff, A. W., Liversedge, S. P., Reichle, E. D., \& Schotter, E. R. (2016). Eye movements in reading and information processing: Keith Rayner's 40 year legacy. Journal of Memory and Language, 86, 1-19.

Clifton, C., Jr., Staub, A., \& Rayner, K. (2007). Eye movements in reading words and sentences. In R. P. G. van Gompel, M. H. Fischer, W. S. Murray, \& R. L. Hill (Eds.), Eye movements: A window on mind and brain (pp. 341-372). Amsterdam: Elsevier.

Forster, K. I., \& Davis, C. (1984). Repetition priming and frequency attenuation in lexical access. Journal of Experimental Psychology: Learning, Memory, and Cognition, 10, 680-698. doi:10.1037/02787393.10.4.680

Forster, K. I., \& Davis, C. (1991). The density constraint on form-priming in the naming task: Interference effects from a masked prime. Journal of Memory and Language, 30, 1-25.

Gordon, P. C., Plummer, P., \& Choi, W. (2013). See before you jump: Full recognition of parafoveal words precedes skips during reading. Journal of Experimental Psychology: Learning, Memory, and Cognition, 39, 633-641.

Grainger, J., \& Jacobs, A. M. (1996). Orthographic processing in visual word recognition: A multiple read-out model. Psychological Review, 103, 518-565. doi:10.1037/0033-295X.103.3.518

Hoedemaker, R. S., \& Gordon, P. C. (2014). It takes time to prime: Semantic priming in the ocular lexical decision task. Journal of Experimental Psychology: Human Perception and Performance, 40, 2179-2197. doi:10.1037/a0037677

Hoedemaker, R. S., \& Gordon, P. C. (2017). The onset and time course of semantic priming during rapid recognition of visual words. Journal of Experimental Psychology: Human Perception and Performance. In press.

Jacoby, L. L., \& Dallas, M. (1981). On the relationship between autobiographical memory and perceptual learning. Journal of Experimental Psychology: General, 110, 306-340. doi:10. 1037/0096-3445.110.3.306

Ledoux, K., Gordon, P. C., Camblin, C. C., \& Swaab, T. Y. (2007). Coreference and lexical repetition: Mechanisms of discourse integration. Memory \& Cognition, 35, 801-815.

Lewellen, M. J., Goldinger, S. D., Pisoni, D. B., \& Greene, B. G. (1993). Lexical familiarity and processing efficiency: Individual differences in naming, lexical decision, and semantic categorization. Journal of Experimental Psychology: General, 122, 316-330. doi:10.1037/ 0096-3445.122.3.316

Liversedge, S. P., Pickering, M. J., Clayes, E. L., \& Branigan, H. P. (2003). Thematic processing of adjuncts: Evidence from an eyetracking experiment. Psychonomic Bulletin \& Review, 10, 667675.

Lowder, M. W., Choi, W., \& Gordon, P. C. (2013). Word recognition during reading: The interaction between lexical repetition and frequency. Memory \& Cognition, 41, 738-751.

Martin-Chang, S. L., \& Gould, O. N. (2008). Revisiting print exposure: Exploring differential links to vocabulary, comprehension, and reading rate. Journal of Research in Reading, 31, 273-284.

Mol, S. E., \& Bus, A. G. (2011). To read or not to read: A meta-analysis of print exposure from infancy to early adulthood. Psychological Bulletin, 137, 267-296.
Moore, M., \& Gordon, P. C. (2015). Reading ability and print exposure: Item response theory analysis of the author recognition test. Behavior Research Methods, 47, 1095-1109. doi:10.3758/s13428014-0534-3

Perfetti, C. A. (1985). Reading ability. New York: Oxford University Press.

Perfetti, C. A. (2007). Reading ability: Lexical quality to comprehension. Scientific Studies of Reading, 11, 357-383.

Rayner, K. (1978). Eye movements in reading and information processing. Psychological Bulletin, 85, 618-660.

Rayner, K. (1998). Eye movements in reading and information processing: 20 years of research. Psychological Bulletin, 124, 372-422. doi: 10.1037/0033-2909.124.3.372

Scarborough, D. L., Cortese, C., \& Scarborough, H. S. (1977). Frequency and repetition effects in lexical memory. Journal of Experimental Psychology: Human Perception and Performance, 3, 1-17. doi:10. 1037/0096-1523.3.1.1

Sears, C. R., Campbell, C. R., \& Lupker, S. J. (2006). Is there a neighborhood frequency effect in English? Evidence from reading and lexical decision. Journal of Experimental Psychology: Human Perception and Performance, 32, 1040-1062. doi:10.1037/00961523.32.4.1040

Sears, C. R., Siakaluk, P. D., Chow, V. C., \& Buchanan, L. (2008). Is there an effect of print exposure on the word frequency effect and the neighborhood size effect? Journal of Psycholinguistic Research, 37, 269-291.

Stanovich, K. E., \& Cunningham, A. E. (1992). Studying the consequences of literacy within a literate society: The cognitive correlates of print exposure. Memory \& Cognition, 20, 51-68.

Stanovich, K. E., \& West, R. F. (1989). Exposure to print and orthographic processing. Reading Research Quarterly, 24, 402-433.

Stanovich, K. E., West, R. F., \& Harrison, M. R. (1995). Knowledge growth and maintenance across the life span: The role of print exposure. Developmental Psychology, 31, 811-826.

Tan, L. C., \& Yap, M. J. (2016). Are individual differences in masked repetition and semantic priming reliable? Visual Cognition, 24, 182200.

Traxler, M. J., Foss, D. J., Seely, R. E., Kaup, B., \& Morris, R. K. (2000). Priming in sentence processing: Intralexical spreading activation, schemas, and situation models. Journal of Psycholinguistic Research, 29, 581-594.

Tulving, E., \& Schacter, D. L. (1990). Priming and human memory systems. Science, 247, 301-306. doi:10.1126/science.2296719

Unsworth, S. J., \& Pexman, P. M. (2003). The impact of reader skill on phonological processing in visual word recognition. Quarterly Journal of Experimental Psychology, 56A, 63-81.

Valentine, T., Bredart, S., Lawson, R., \& Ward, G. (1991). What's in a name? Access to information from people's names. European Journal of Cognitive Psychology, 3, 147-176.

Valentine, T., Moore, V., Flude, B. M., Young, A. W., \& Ellis, A. W. (1993). Repetition priming and proper name processing. Do common names and proper names prime each other? Memory, 1, 329349.

Van Petten, C., Kutas, M., Kluender, R., Mitchiner, M., \& McIsaac, H. (1991). Fractionating the word repetition effect with event-related potentials. Journal of Cognitive Neuroscience, 3, 131-150.

Yap, M. J., Tse, C. S., \& Balota, D. A. (2009). Individual differences in the joint effects of semantic priming and word frequency revealed by RT distributional analyses: The role of lexical integrity. Journal of Memory and Language, 61, 303-325. 